\title{
Vertragswettbewerb zwischen Leistungserbringern in der Integrierten Versorgung
}

Modelle integrierter Versorgung werden uns meist als Leuchtturmprojekte präsentiert. Doch echte Leuchttürme haben den Regelbetrieb eingestellt und dienen nur noch als unter Denkmalschutz gestellte Touristenattraktion. Die ständigen Gesundheitsreformen und die damit verbundene fehlende Planungssicherheit sowie intransparente Entscheidungen auf Krankenkassenseite halten viele Leistungserbringer davon ab, zukunftsweisende Projekte integrierter Versorgung zu entwickeln.

\section{Karsten Scholz}

Das Krankenversicherungsrecht wird bisher von Kollektivverträgen bestimmt. Charakteristikum der letzten Gesundheitsreformen war jeweils ein deutlicher Schritt hin zu einem Einzelvertragssystem. Dabei wird der Integrierten Versorgung ein besonderes Entwicklungspotential beigemessen, was ihr seit dem GKV-WSG allerdings durch die besondere ambulante ärztliche Versorgung nach § 73b SGB V streitig gemacht werden könnte. Gleichzeitig wird die Fusion von Krankenkassen erleichtert und auf diese Weise die Verhandlungsmacht zugunsten der Krankenkassen verschoben.

Der Gesetzgeber hat durch die Anschubfinanzierung und den nunmehr bis Ende 2008 verlängerten Dispens vom Grundsatz der Beitragssatzstabilität mit dem GMG 2004 auch auf Leistungserbringerseite Anreize zum Abschluss von Verträgen über eine Integrierte Versorgung geschaffen. Seinerzeit anerkannte die Gesetzesbegründung, dass die Entwicklung und der Aufbau solcher Strukturen und interner Vergütungssysteme zusätzliche Kosten verursacht, die von den Krankenkassen mitfinanziert werden können. Das gilt allerdings nur dann, wenn es tatsächlich zum Vertragsabschluss kommt. Viele Leistungserbringer haben daher in Hoffnung auf einen Vertragsabschluss vergeblich in den Aufbau von zunächst informellen Strukturen investiert, ohne dass diese Kosten refinanziert wurden.

Mit dem GKV-WSG ist das Gesetz in der Frage der Erstattung zusätzlicher Anlaufkosten jedoch widersprüchlich geworden. § 53 Abs. 3 SGB V sieht nunmehr vor, dass die Krankenkasse in ihrer Satzung einen besonderen Tarif für Versicherte vorzusehen hat, die ihre Teilnahme

Dr. jur. Karsten Scholz. Justiziar der Ärztekammer Niedersachsen, Lehrbeauftragter an der Leizniz-Universität Hannover und der Fachhochschule Osnabrück an der integrierten Versorgungsform erklären. Es können Prämienzahlungen oder Zuzahlungsermäßigungen vorgesehen werden. Nach § 53 Abs. 9 SGB V muss sich der Wahltarif selbst tragen, wobei eine mittel- oder langfristige Betrachtung in Bezug auf Einsparungen und Effizienzsteigerungen ausgeschlossen ist. Das hat zur Folge, dass nur noch kurzfristig Kosten senkende Verträge abgeschlossen werden können und die Einsparungen zudem so hoch sein müssen, dass dadurch auch die mit der Einführung der Wahltarife verbundenen höheren Verwaltungskosten der Krankenkassen getragen werden können.

Der Gesetzgeber vertritt mithin die durch empirische Erhebungen in keiner Weise gedeckte These, dass Integrierte Versorgungsmodelle flächendeckend sowohl die medizinische Versorgung der Patienten verbessern als auch Wirtschaftlichkeitsreserven erschließen. Es wird damit die ebenfalls in der Gesetzesbegründung zum GMG zu findende Position zurück genommen, dass eine fachübergreifende Versorgung in einem medizinischen Versorgungszentrum bei besserer Patientenversorgung jedenfalls initial mit höheren Kosten verbunden sein kann.

Auch volkswirtschaftlich ist die These nicht belegt, dass ein Einzelvertragssystem effizienter ist. Insofern lohnt ein Seitenblick auf den Öffentlichen Personennahverkehr (ÖPNV). Die Hans-Böckler-Stiftung hat im November 2006 ihr Projekt „ÖPNV zwischen Ausschreibungswettbewerb und Direktvergabe - eine vergleichende Studie zu Produktions- und Transaktionskosten“ vorgestellt. Die Situation ist meines Erachtens durchaus vergleichbar, denn der ÖPNV war bislang durch monopolartige Strukturen öffentlicher Unternehmen geprägt.

Die Studie kommt zu dem auch im Gesundheitswesen zu beobachtenden Ergebnis, dass allein die Diskussion um Wettbewerbsstrukturen erhebliche Veränderungspro- 
zesse, insbesondere aber Marketingaktivitäten ausgelöst hat. Die der Nahverkehrsbehörde als Analogon zu den Krankenkassen übertragene Steuerungsfunktion stößt ebenfalls sinnvolle Prozesse an, hat dort aber zu einem personellem und sächlichen Mehrbedarf geführt, unter anderem für die Festlegung und Kontrolle von Qualitätskriterien. Auf Seiten der Anbieter von Verkehrsleistungen entstehen erhebliche Kosten im Stadium der Vorbereitung und Begleitung des Bieterverfahrens, im übrigen eine Tatsache, die auch im Rahmen der Privatisierung der niedersächsischen Landeskrankenhäuser zu beobachten war, über die aber kaum diskutiert worden ist. Zusammenfassend kommt die Studie der Hans-Böckler-Stiftung unter diversen Prämissen zu der Annahme, dass sich das Wettbewerbsmodell erst lohnt, wenn der Aufwand für die Verkehrsleistung je vergebenen Kilometer bezogen auf das Jahr 2004 um 40 bis 50\% sinkt. Diese Einsparungen können jedoch nur erreicht werden, wenn sich das Vergütungsniveau der Beschäftigen deutlich verringert.

In einem Einzelvertragssystem besteht mithin die Tendenz, dass die Leistungserbringer höhere Anteile ihrer aus Sozialversicherungsbeiträgen finanzierten Vergütung für den Einkauf von Beratungsdienstleistungen höherer Art aufgewenden müssen. Diese wiederum kommen nur zum Teil Patienten zugute, indem andere Professionen z. B. den Aufbau sektorenübergreifender Behandlungspfade unterstützen. Erhebliche Kosten werden für die Inanspruchnahme der rechts- und steuerberatenden Berufen und zunehmend auch der Werbewirtschaft aufgewandt. Die Drucksache 16/3100, mit der das GKV-WSG seitens der Koalitionsfraktionen in den Deutschen Bundestag eingebracht wurde, enthält dazu unter dem Stichwort Finanzielle Auswirkungen bezeichnenderweise keine Ausführungen. Die politische Diskussion betrifft allenfalls die mit der Gesetzgebung verbundenen Bürokratiekosten, nicht jedoch die Aufwendungen der Leistungserbringer für ihre Neupositionierung auf dem Gesundheitsmarkt.

Das System punktueller Einzelverträge, auf deren Abschluss kein Rechtsanspruch besteht und bei denen vermeintliche Diskriminierungen angesichts der Vertraulichkeit, denen die Verträge unterliegen, nur eingeschränkt überprüfbar sind, führt dazu, dass Entwicklungspotentiale nur eingeschränkt ausgeschöpft werden. Konzepte könnten besser werden, wenn nicht nur die Krankenkassen sondern auch die Leistungserbringerseite bereits in einem frühen Stadium der Entwicklung neuer Versorgungsstrukturen andere Professionen einbinden könnte. Da jedoch bis zum Vertragsschluss unklar ist, ob derartige Aufwendungen von Kassenseite getragen werden, werden externe Berater in der ersten Projektphase häufig nur zurückhaltend und stärker erst dann einbezogen, wenn die Grundkonzeption bereits nicht mehr modifizierbar ist. Dieses führt auf Leistungserbringerseite zu Wettbewerbsvorteilen von Krankenhauskonzernen.

Ein Leistungserbringer wird sich nur dann auf den Vertragswettbewerb in der Integrierten Versorgung einlassen, wenn er davon mutmaßlich finanziell profitiert. Die
Höhe der Vergütung ist dafür nur einer, wenn auch der wesentlichste von mehreren Parametern. Für Krankenhäuser und Rehabilitationskliniken spielt die Sicherung von Einweiserbeziehungen eine erhebliche Rolle. Da es § 31 der Berufsordnungen aller Landesärztekammern verbietet, für die Zuweisung von Patienten ein Entgelt oder andere Vorteile zu versprechen oder zu gewähren und darüber geschlossene Verträge gem. § 134 BGB nichtig sind, dürfen die Integrationsverträge nicht dazu missbraucht werden, die Vergütung abweichend vom tatsächlichen Leistungsaufwand der Beteiligten zu verteilen. Auch das legitime Ziel, eine durchgängige gleichmäßige Belegung der Klinik zu erreichen und damit Effizienzgewinne zu erzielen, darf nicht zulasten derjenigen Patienten gehen, die aus medizinischen Gründen sofortiger Behandlung bedürfen.

Kostenvorteile ergeben sich für die Leistungserbringer meist daraus, dass die Dokumentation sektorenübergreifend aufeinander abgestimmt wird und Nachfragen von zuweisenden und nachbehandelnden Kollegen sowie von Krankenkassen deutlich abnehmen. Die Möglichkeiten, Bürokratiekosten in diesem Bereich einzusparen, werden allerdings häufig überschätzt. Der Behandlungsverlauf und jegliche Besonderheiten sind aus Gründen des zivilen Haftungsrechts auch weiterhin sorgfältig zu dokumentieren. Durch die Verzahnung multilokaler Einheiten ergeben sich zudem weitergehende Organisationspflichten, welche die ständige Überprüfung des Prozesses erfordern. Schließlich müssen in aller Regel die Investitionskosten im EDV-Bereich gegengerechnet werden.

In finanzieller Hinsicht sind für den Leistungserbringer auch die Vertragsdauer und die Kündigungsmöglichkeiten auf Krankenkassenseite von erheblicher Bedeutung. In den mir bekannten Verträgen war die Kündigungsfrist sehr kurz bemessen. Jede weitere Gesundheitsreform kann zudem zu einem Wegfall der Geschäftsgrundlage des Integrationsvertrages führen. Das erschwert die Beschaffung von Fremdkapital, was im Übrigen auch ganz generell bei einem Übergang vom Kollektivvertrags- zum Einzelvertragssystem gelten dürfte. Zusätzliche Aufwendungen entstehen dem Leistungserbringer, wenn die Vertragspartner wie häufig vereinbaren, dass er noch weitere Qualifikationen zu erwerben hat. Die damit verbundene höhere Strukturqualität kann gerade bei Vertragsärzten eine Nachfolgeregelung im Nachbesetzungsverfahren erschweren, weil die Krankenkasse von einem Arzt, der in den Vertrag eintritt, in aller Regel erwartet, dass er die vom Vorgänger erfüllte Qualifikation bereits mitbringt.

Der Leistungserbringer muss sich vor Beginn der Verhandlungen überlegen, ob er zur Übernahme der Budgetverantwortung bereit ist. Dabei wird er sich in Erinnerung zu rufen haben, dass die bis dahin geringe Risikostreuung in den 1880er Jahren ein ganz wesentlicher Grund für das Entstehen größerer Krankenkassen war. Auch könnten Krankenkassen bestrebt sein, die mit der vertragsarztärztlichen Vergütungsreform des Jahres 2009 verbundene Übernahme des Morbiditätsrisikos durch Abschluss entsprechender Einzelverträge abzuwenden. Ent- 
sprechendes gilt, wenn sich der Leistungserbringer zu einer leitliniengerechten Behandlung verpflichtet. Es bedarf einer Regelung, wer das finanzielle Risiko höherer Kosten bei Einbeziehung neuer medizinischer Methoden bzw. Erkenntnisse trägt.

Wohlüberlegt will sein, ob die Krankenkasse bei der Honorarverteilung wie seit dem GMG bei der vertragsärztlichen Honorarverteilung mitbestimmen soll oder die Leistungserbringer nur untereinander für eine gerechte Verteilung sorgen wollen. Je größer deren Verbund ist, desto größerer Aufwand muss hierfür betrieben werden und desto bessere Konfliktlösungsmechanismen werden benötigt. Die Krankenkasse wird auch auf die Übernahme einer Budgetverantwortung für veranlasste Leistungen dringen und die verordnenden Leistungserbringer dafür im Gegenzug ggf. an Einsparungen beteiligen wollen. Die Einbeziehung des Pflegebereichs durch die jüngste Gesundheitsgesetzgebung wird den Fokus hierauf noch stärker als in der Vergangenheit richten. Speziell bei populationsbezogenen integrierten Versorgungen muss zudem auf eine exakte Beschreibung des Versorgungsauftrags geachtet werden. Nur so können die Krankenkassen sicherstellen, dass Doppelabrechnungen unterbleiben und Leistungserbringer erreichen, dass alle Leistungen tatsächlich vergütet werden.

Für den Leistungserbringer stellt sich die weitere Frage, ob er mehr oder weniger allein oder gemeinsam mit anderen auf die Krankenkasse zugeht oder Dritte für ihn verhandeln und die Verträge abschließen lässt. Anders als die Krankenhäuser haben die Vertragsärzte bislang weitgehend keine Erfahrungen in Verhandlungen mit Krankenkassen. Das GKV-WSG ermöglicht es daher den Kassenärztlichen Vereinigungen in § 77a SGB V, ihren Mitgliedern durch organisatorisch ausgegliederte Dienstleistungsgesellschaften gegen besonderes Entgelt Beratungsleistungen anzubieten und damit das Verhandlungsungleichgewicht auszugleichen. Die Gesellschaften treten in Konkurrenz zu sogen. Managementgesellschaften, welche eine integrierte Versorgung durch berechtigte Leistungserbringer anbieten, § 140b Abs. 1 Nr. 2 SGB V.

Diese Strukturen kommen den Krankenkassen entgegen, die sich Ansprechpartner mit Verhandlungsmandat und Managementkompetenzen wünschen. Andererseits unterliegen die Gesellschaften nicht den Beschränkungen des öffentlichen Rechts. Im Rahmen der integrierte Versorgung findet daher bei näherer Betrachtung nicht nur ein Qualitätswettbewerb zwischen den Leistungserbringern sondern auch Wettbewerb um das Management von Gesundheitsdienstleistungen statt. Neben den Kollektivvertragspartnern, denen allerdings keine Vertragsabschlusskompetenz im Rahmen der integrierten Versorgung zusteht, konkurrieren vertragsarztgetragene Verbünde und Genossenschaften, „Vollsortimenter“, d.h. Krankenhauskonzerne mit eigenen medizinischen Versorgungszentren sowie Beratungsunternehmen miteinander, die im Begriff sind, Marken aufzubauen.

Der Gesetzgeber erhofft sich hierdurch, ebenso wie durch die Etablierung unternehmerisch geführter medizi- nischer Versorgungszentren, das Erschließen von Wirtschaftlichkeitsreserven. Er kommt damit zugleich einem Wunsch vieler junger Ärztinnen und Ärzte nach, die sich zunächst auf die medizinische Versorgung ihrer Patienten konzentrieren wollen und daher die Angestelltentätigkeit einer eigenen Niederlassung vorziehen. Dabei verletzt der Gesetzgeber allerdings mangels sachlichen Grundes die grundgesetzlich geschützte Berufsfreiheit, wenn er Ärzten zwar durch bloße Erklärung ermöglicht, auf ihre Zulassung zu verzichten, um sich in einem medizinischen Versorgungszentrum oder bei einem anderen Vertragsarzt anstellen zu lassen, es ihnen aber später verwehrt, ohne weiteres (Mit-) Inhaber bzw. Gesellschafter der Einrichtung zu werden.

Ob sich der Vertragsarzt allein oder gemeinsam mit Anderen dem Vertragswettbewerb stellt, hängt von der Wettbewerbssituation in seiner Fachgruppe ab. Fehlt es an Nachwuchs, kann der Einzelne höhere Preise aushandeln. Werden bestimmte Leistungen, vor allem auch im internationalen Vergleich, in Deutschland besonders häufig erbracht, werden die Krankenkassen bestrebt sein, nur mit einzelnen Anbietern Verträge zu schließen und damit das Angebot zu begrenzen. Damit gehen sie allerdings auch erhebliche eigene Haftungsrisiken ein. Beispielsweise ist dem Autor bekannt, dass eine Krankenkassen einen Integrationsvertrag mit einem Arzt stark bewirbt, dem der Krankenhausträger an seiner früheren Wirkungsstätte ein Operationsverbot erteilt hatte.

Zunächst mit hoher Präsenz wurden vielerorts Praxisnetze gegründet. Die Startphase zieht sich meistens lange hin, was nicht zuletzt am deutschen Perfektionsstreben liegt. Dadurch lässt die Motivation Vieler nach, so dass das Vorhaben nur noch von einem Teil der Mitglieder voll unterstützt wird. Das macht es dem erst nach Vertragsabschluss bezahlbaren und daher erst in dieser Phase gewonnenen Manager schwer, die gesteckten Ziele zu erreichen.

Gegenüber solchen Strukturverträgen haben Integrationsverträge den scheinbaren Vorteil, dass durch die Beteiligung von Krankenhäusern auf vorhandene Managementstrukturen zurückgegriffen werden kann. In der Realität müssen diese sich jedoch erst das Know-how für den ambulanten Versorgungssektor erwerben und dafür externe Beratungsleistungen in Anspruch nehmen. Es werden zudem Beiräte sowie Projekt- und Steuerungsgruppen gebildet, deren Kompetenzen beschrieben werden müssen und die zunächst zusätzliche Arbeitskraft binden.

Ohne dass dieses den Beteiligten zunächst bewusst wäre, gründen die auf Leistungserbringerseite Beteiligten schon in einem frühen Stadium im Regelfall eine Gesellschaft bürgerlichen Rechts nach den §§ 705ff. BGB. Sofern später auf keine Managementgesellschaft zurückgegriffen wird, übernimmt diese die Gewähr für die standardgemäße Erfüllung der organisatorischen, betriebswirtschaftlichen, medizinischen und medizintechnischen Voraussetzungen des vereinbarten Versorgungsauftrags. Das hat zur Folge, dass alle Beteiligten 
auch für Fehler der jeweils Anderen jedenfalls im Außenverhältnis mit ihrem Privatvermögen haften. Daher zögern insbesondere Vertragsärzte mit der Vertragsunterzeichnung, wenn am Integrationsvertrag Krankenhäuser beteiligt sind. Durch den Außenauftritt des Integrationsverbundes und die in Imagebroschüren dargestellte verbesserte Kommunikation sowie das optimierte Nahtstellenmanagement übernimmt die Gemeinschaft zudem gegenüber den Patienten zusätzliche Pflichten, was die Haftungssituation verschärft.

Die Gewährsübernahme führt insbesondere bei populationsbezogenen Integrationsverträgen häufig auch zur Abhängigkeit von einzelnen Leistungserbringern. Nur eingeschränkt kann für den Fall vorgesorgt werden, dass der beteiligte Radiologe plötzlich stirbt, seine Zulassung verliert oder wegen der Verletzung von Treuepflichten gegenüber anderen Gesellschaftern ausgeschlossen werden muss. Der Vertrag sollte daher regeln, ob die Krankenkasse zumindest einen Teil dieses Risikos übernimmt. Der Verbund als privatrechtlich organisierte Gesellschaft ist andererseits nicht völlig frei, wen er aufnimmt. Insofern ist die Rechtsprechung zu beachten, nach der ein Taxiunternehmer unter bestimmten Voraussetzungen Anspruch auf Aufnahme in den Trägerverein der Taxizentrale hat.

Zur Zeit gilt für den Bereich der Integrierten Versorgung: Wer die beste Idee hat, kann mit einem Vertragsabschluss belohnt werden. Die beste Idee kann aber auch andernorts verwirklicht werden. Weil den Leistungserbringern die genauen Vertragsinhalte verborgen bleiben, sind ihre Konzepte nur unzureichend gegen die Verwertung Dritter an anderen Stellen der Republik geschützt. Es ist daher bedenklich, dass die Krankenkassen, obwohl es sich um Körperschaften öffentlichen Rechts handelt, vom Anwendungsbereich des Informationsfreiheitsgesetzes ausgenommen sind. Jedenfalls bei Standardverträgen wie z.B. im Bereich der Katarakt-Operationen ist zu fordern, dass die Vertragsabschlüsse Ergebnis eines transparenten und Rechtsschutzmöglichkeiten eröffnenden Vergabeverfahrens sind.

Dem kann auch nicht entgegengehalten werden, die Patienten übten eine entsprechende Kontrollfunktion aus, weil sie sich mit der Teilnahme am Integrationsvertrag jeweils einverstanden erklären müssten. Den Patienten werden nur die Grundzüge des Vertrages erläutert; einsehen können auch sie ihn nicht. Gleichwohl zeigt die bisherige Erfahrung, dass Patienten, die sich nicht einschreiben, in gleicher Art und Weise wie Patienten behandelt werden, die den Wahltarif wählen. Für Leistungserbringer wäre es viel zu aufwändig, zwischen beiden Patientengruppen zu unterscheiden. Allerdings führen Integrationsverträge manchmal auch zu einer Dreiklassenmedizin. So entgegnete mir der Vertreter einer großen Ersatzkasse auf meinen Hinweis, offenbar dürfe der Vertragsarzt keine Weiterbildungsassistenten in die Behandlung einbeziehen: „Der kann doch AOK-Patienten operieren."

Die Integrierte Versorgung ist durch das GMG und den mit ihr verbundenen ersatzlosen Wegfall der Rahmenvereinbarung stark gefördert worden. Ich teile die Einschätzung, dass das Gesetz mit Blick darauf, dass es um die Verwendung öffentlicher Gelder geht, ein Untermaß an Regelungen enthält. Vor allem fehlt die Verpflichtung der Krankenkassen, erfolgreiche Projekte nach Abschluss in die Regelversorgung zu überführen. Solange kann nicht ausgeschlossen werden, dass deren finanzielle Überlegungen bei der Auswahl der Projekte stärkeres Gewicht als die mit dem jeweiligen Projekt tatsächlich verbundene Verbesserung der Patientenversorgung haben. Die geringe Regelungsdichte lässt zudem die Frage aufkommen, ob die hohe Kontrolldichte im Regelversorgungsbereich noch erforderlich ist.

Im Jahr 2030 wird die Frankfurter Allgemeine Zeitung auf die Jahre 2004 bis 2008 zurückblicken. Wir können auf das Fazit gespannt sein. Ich halte zwei Varianten der Berichterstattung für denkbar. Die erste lautet: „Ulla Schmidt hat geschafft, dass auch in Deutschland endlich die Sektorengrenzen überwunden wurden und patientenorientierte, leitliniengerechte und qualitätsgesicherte Behandlungspfade bestehen." Ich halte aber auch folgenden Text für möglich: „Anfang des Jahrtausends ebnete eine sozialdemokratische Gesundheitsministerin den Weg für ein Oligopol börsennotierter Gesundheitskonzerne in Deutschland." 